\title{
Multiscale Segmentation Techniques for Textile Images
}

\author{
Xiqun Lu \\ College of Computer Science, Zhejiang University \\ Hangzhou, 310027,
}

China

\section{Introduction}

In this chapter we focus on image segmentation techniques for some very special images textile images. They are generated from the color halftoning technique in textile and printing production lines. In contrast with natural color images, textile images have some very distinctive features: (1) generally there are a few dominant colors in a textile image, whereas there may exist hundreds of significant colors in a natural color image; (2) in the textile industry designers combine threads of different colors, thickness, and densities to produce the visual impression of other colors (the color halftoning technique in textile printing). The fabric texture structure - texture noise - has a great influence on the colors' appearance in textile images, which makes the color segmentation of textile images a very difficult problem [1].

A typical textile image is shown in Fig.1. According to human visual observation, there are about six dominant colors in the textile image, but when the image is enlarged to the pixellevel, we will find that there are many different colors in perceived uniform color regions as shown in Fig.1. (a) and (b). Four histograms of the spatial variation magnitudes of the four uniform and edge blocks (as shown in Fig.1 (a), (b), (c) and (d)) are illustrated in Fig.2 (Here the spatial variation magnitude is computed as the added value of the two absolute forward differences along the horizontal and vertical directions on the luminance component of each

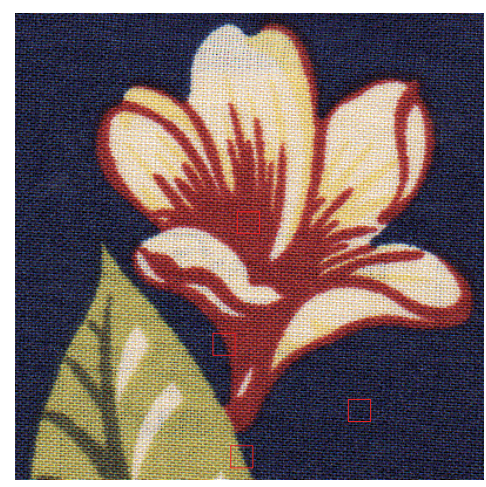

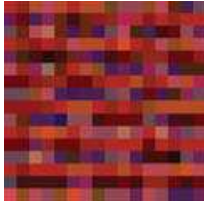

(a) Uniform red area

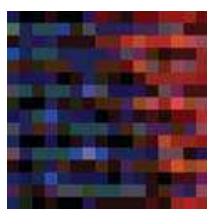

(c) Edge area I

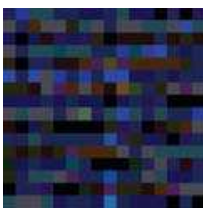

(b) Uniform blue area

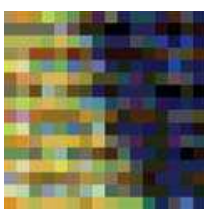

(d) Edge area II

Fig. 1. A typical textile image 


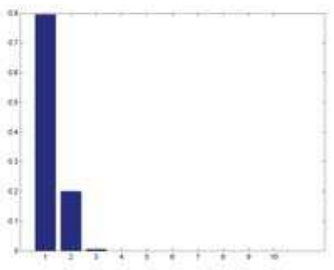

(a) Uniform red area

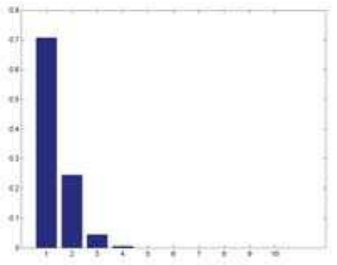

(c) Edge area I

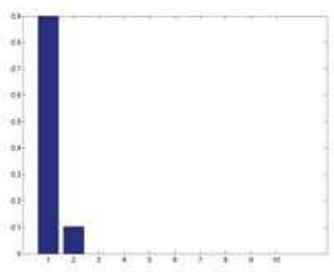

(b) Uniform blue area

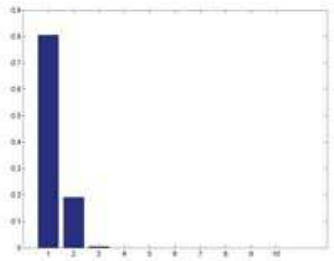

(d) Edge area II

Fig. 2. Histograms of the spatial variation magnitudes of uniform areas and edge areas in the textile image

block). From these histograms, we can see that it is very difficult to distinguish the variations caused by genuine spatial edges from those caused by texture noise.

Some distinctive properties of texture noise are compared with those of white Gaussian noise in [2]. Fig.3. (a) shows a $16 \times 16$ texture noise block extracted from the textile image as shown in Fig.1. Fig.3. (d) is a $16 \times 16$ white noise block generated by the "randn" function in MatlabTM. Texture noise is clearly non-Gaussian distributed, and has highly correlative spatial structure.

Any image segmentation algorithm that independently classifies each pixel in the image plane is unlikely to perform well for textile images since there is insufficient information to make a good decision. So many segmentation algorithms divide the image into arbitrary blocks, and classify each block independently. However, if the block size is too small, discriminating among similar textures may be difficult. Alternatively, if the block size is too large, regions of differing textures may be lost. In either case, the resulting boundaries will not be accurate since there is no reason to believe that the actual boundaries occurred along the block boundaries [3]. Multiscale image segmentation approaches [1,3-14] have been proven efficient to integrate both image features and contextual information to classify a region in an image differently from its surroundings if there is sufficient statistical evidence to justify a distinct region regardless of size, and refine the segmentation results recursively between different scales. The number of important contributions in this area is so great that just listing all of them would more than exhaust the page budget of this chapter (for example, the bibliography in [4] is 8 pages in a two-column format). So in Section 2 we review a few representative multiscale image segmentation techniques which developed over the past decades. In Section 3 we test some of the benchmark multiscale image segmentation techniques on textile images, and compare the experimental results analytically. We finally give some concluding remarks in Section 4. In the following sections, we will follow the convection of using upper-case letters for random variables and lower-case letters for their realizations. 


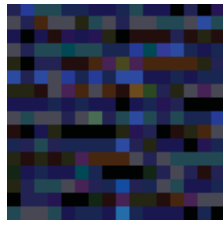

(a) Texture noise

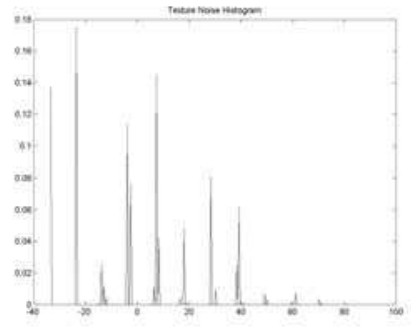

(b) PDF of texture noise

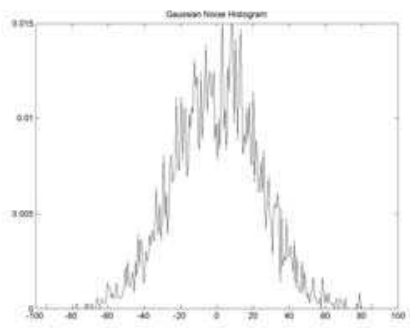

(e) PDF of white noise

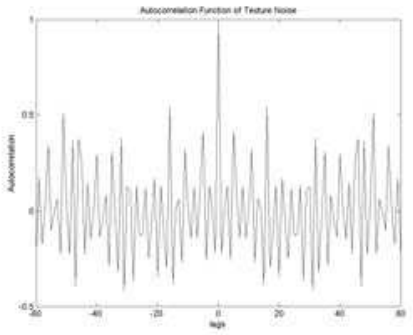

(c) Autocorrelation function of texture noise

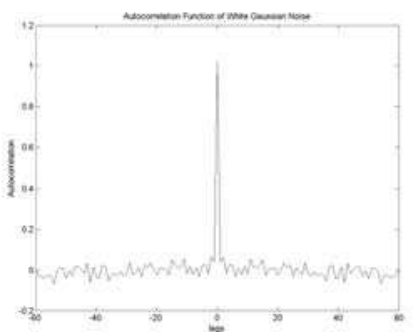

(f) Autocorrelation function of white noise

Fig. 3. Distinctive properties of texture noise

\section{Multiscale image segmentation techniques}

\subsection{Problem statement and notations}

The image segmentation problem can be considered as the process of inferring the "best" configuration of labelling $X$ from the observed image data $Y=y$, and both random fields $X$ and $Y$ are defined on a rectangular gird $S$. Each random variable of $X$ takes its values from a finite set of class labels $\Lambda=\left\{\begin{array}{lll}1 & \cdots & K\end{array}\right\}$, where $K$ is the total number of classes. The standard Bayesian formulation of this inference problem consists in minimizing the expectation of some cost function $C$, given the data

$$
\hat{x}=\underset{x}{\arg \min } E(C(X, x) \mid Y=y)
$$

Where $C(X, x)$ is the cost of estimating the true segmentation $X$ by an approximate segmentation $x$.

The MAP (Maximum a Posteriori) estimator is an solution of (1) if we use the cost functional of $C(X, x)=1$ whenever any pixel is incorrectly classified. The MAP estimator aims at maximizing the probability that all pixels will be correctly classified. It is well known that the MAP estimator is excessively conservative, and appear even more inappropriate for the estimation of multiscale Markov random fields [6].

An alternative criterion to the MAP estimation is to minimize the probability of classification error, the Maximization of the Posterior Marginals (MPM) estimator, which associates to each cite the most probable value given all the data 


$$
\forall s \in S, \quad \hat{x}_{s}=\underset{x_{s} \in \Lambda}{\arg \min } P\left(x_{s} \mid Y=y\right)
$$

The MPM estimator results in high computational complexity since each of these functions should be obtained by simultaneously integrating out all $x_{t}(t \neq s)$ [5]. Usually in real situations, it is unfeasible to compute these posterior marginals $P\left(x_{s} \mid y\right)$ exactly since the original joint distribution $P(x, y)$ is unavailable or it is intractable to specify the joint distribution for each possibility.

\subsection{Sequential MAP ${ }^{[6]}$}

Although the MPM criterion seems to be more appropriate than the MAP criterion, both the MPM and MAP cost functions do not take into account the location of estimation errors in a hierarchical quadtree structure. Bouman and Shapiro introduced the following cost function in [6]

$$
C_{S M A P}(X, x)=\sum_{n=0}^{L} 2^{n-1}\left(1-\prod_{i=n}^{L} \delta\left(X^{(i)}, x^{(i)}\right)\right)
$$

At each scale $n$, the segmentation or labeling is denoted by the random field $X^{(n)}$, and the set of lattice points is denoted by $S^{(n)}$. This sequential MAP (SMAP) cost function is to sum up the segmentation errors from multiple scales together. The SMAP estimator aims at minimizing the spatial size of errors.

The multiscale image model proposed in [6] is composed of a series of random fields at multiple scales. Each scale has a random field of image feature vectors $Y^{(n)}$, and a random field of class labels $X^{(n)}$. We denote an individual sample at scale $n$ by $y_{s}^{(n)}$ and $x_{s}^{(n)}$, where $s$ is the position in the 2-D lattice $S^{(n)}$. Markovian dependencies are assumed across scales to capture interscale dependencies of multiscale class labels, and the SMAP recursion can be estimated in a fashion of coarse-to-fine

$$
\hat{x}^{(n)}=\underset{x^{(n)}}{\arg \max }\left\{\log p_{y^{(n)} \mid x^{(n)}}\left(y^{(n)} \mid x^{(n)}\right)+\log P_{x^{(n)} \mid x^{(n+1)}}\left(x^{(n)} \mid \hat{x}^{(n+1)}\right)\right\}
$$

The two terms in (4) are the likelihood function of the image feature $y^{(n)}$ and the contextbased prior knowledge from the next coarser scale, respectively. Specifically, the quadtree pyramid developed in [6] is to capture interscale dependencies of multiscale class labels regarding the latter part of (4). But a big problem with the quadtree pyramid is that spatially adjacent samples may not have a common parent sample at the next coarser scale which may result in discontinuous boundaries. Therefore a more generalized pyramid graph model was introduced in [6] where each sample has multiple parents at the next coarser scale. However, this pyramid graph also complicates the computation of likelihood functions, and the coarse-to-fine recursion of (4) has to be solved approximately.

Based on the same framework, a trainable context model for multiscale Bayesian segmentation was proposed in [7], where the contextual behavior can be trained off-line by providing image data the corresponding ground truth segmentations. Then the segmentation can be accomplished efficiently via a single fine-to-coarse-to-fine iteration through the pyramid. 


\subsection{Wavelet-domain HMT ${ }^{[8]}$}

A distinct context-based Bayesian segmentation algorithm was proposed in [8] where the context model is characterized by a context vector $v^{(n)}$ derived from a set of neighboring samples $(3 \times 3)$ at the next coarse scale. It is assumed that, given $y_{s}^{(n)}$, its context vector $v_{s}^{(n)}=\left\{\begin{array}{ll}x_{p s}^{(n)} & x_{l s}^{(n)}\end{array}\right\}$ can provide supplement information regarding $x_{s}^{(n)}$, where $x_{p s}^{(n)}$ denotes the class label of its parent sample, and $x_{l s}^{(n)}$ denote the dominant class label of the $3 \times 3$ samples at the next coarser scale. Given $v_{s}^{(n)}, x_{s}^{(n)}$ is independent with all other class labels. In particular, the contextual prior $p_{x^{(n)} \mid v^{(n)}}(c \mid u)$ is involved in the SMAP estimation which has the same purpose as the second term in (4), and it can be estimated by maximizing the following context-based mixture model likelihood as

$$
f\left(y^{(n)} \mid v^{(n)}=u\right)=\prod_{s \in S^{(n)}} \sum_{c=1}^{K} p_{x^{(n)} \mid v^{(n)}}\left(x_{s}^{(n)}=c \mid \hat{v}_{s}^{(n)}=u\right) f\left(y_{s}^{(n)} \mid c\right)
$$

where the likelihood function $f\left(y^{(n)} \mid x^{(n)}\right)$ is computed by using the wavelet-domain hidden Markov model (HMT) proposed in [9]. An iterative Expectation Maximization (EM) training algorithm was developed in [8] to approach the above problem.

The HMT is a tree-structure model in the wavelet-domain to characterize the joint statistics of wavelet coefficients across scales. In order to perform multiscale segmentation, an image is recursively divided into four sub-images of same size $J$ times, and a pyramid is constructed with $J$ scales. A block $y^{(n)}$ at scale $n$ is associated with three wavelet subtrees in three subbands as, $\left\{\begin{array}{lll}T_{L H}^{(n)} & T_{H L}^{(n)} & T_{H H}^{(n)}\end{array}\right\}$. The computation of the model likelihood of $f\left(y^{(n)} \mid \theta\right)$ is a realization of the HMT model $\theta$ and is obtained by

$$
f\left(y^{(n)} \mid \theta\right)=f\left(T_{L H}^{(n)} \mid \theta_{L H}\right) f\left(T_{H L}^{(n)} \mid \theta_{H L}\right) f\left(T_{H H}^{(n)} \mid \theta_{H H}\right)
$$

Where it is assumed that the three DWT subbands are independent and each one in (6) can be estimated based on a close formula proposed in [9].

An improved wavelet-domain hidden Markov model, HMT-3S [10], was developed to capture the wavelet coefficients dependencies not just across scales, but across subbands as well, where the three DWT subbands are grouped into one quadtree structure. It is worth noting that two-state GMMs (Gaussian Mixture Model) in HMT [8] are still used to characterize the DWT marginal statistics, and there will be eight states in a node of HMT-3S. Thus, HMT-3S is parameterized by

$$
\theta_{H M T-3 S}=\left\{p_{J}(u), \varepsilon_{j, j-1}^{u, v}, \sigma_{b, j, h}^{2} \mid b \in B, j=1, \cdots J ; u, v=0, \cdots, 7 ; h=0,1\right\}
$$

Where $B=\left\{\begin{array}{lll}L H & H L & H H\end{array}\right\}$, and $\varepsilon_{j, j-1}^{u, v}$ is the transition probability of the Markov chain from scale $j$ to scale $j-1$. The EM training algorithm in [8] can be straightforwardly extended to the eight-state HMT-3S. 


\subsection{Joint multi-context and multiscale HMT-3S ${ }^{[12]}$}

Most multiscale segmentation algorithms adopt SMAP estimator and mainly consider interscale dependencies of multiscale class labels by assuming Markovian dependencies across scales, and intrascale dependencies are not considered due to the fact that the noncausal structure usually requires extra iterative training process.

However, the characterization of intrascale dependencies is useful for boundary localization [11]. Based on three numerical segmentation criteria: 1) $P_{a}$ the percentage of pixels which are correctly classified; 2) $P_{b}$ the percentage of boundaries that are coincided with the true ones; 3) $P_{c}$ the percentage of true boundaries that can be detected, Fan and Xia quantified the segmentation performances of five contextual models in [11]. And show that interscale context models (context-1 and context-2) may favor $P_{a}$ by encouraging the homogeneity of texture classification across the scales of the pyramid, and the intrascale context model (context-5) may help $P_{c}$ by being sensitive to texture boundaries within a scale. As hybrid context models (context-3 and context-4) may provide high $P_{b}$ by appropriately balancing both interscale dependencies and intrascale dependencies of multiscale class labels into the MAP estimation. It is shown that none of the five context models can work well singly [11]. Since a single context model is unable to provide high accuracy for both texture classification and boundary localization, Fan and Xia proposed a joint multi-context and multiscale (JMCMS) approach to Bayesian segmentation in [12] which reformulates (1) as a multi-object optimization as

$$
\begin{aligned}
& \hat{x}=\underset{x}{\arg \min } E\left(C_{S M A P}(X, x) \mid Y=y_{1}\right) \\
& \vdots \\
& \hat{x}=\underset{x}{\arg \min } E\left(C_{S M A P}(X, x) \mid Y=y_{z}\right)
\end{aligned}
$$

Where an image $y$ can be represented as multiple $(Z)$ copies characterized by distinct context models, i.e., $\left\{y_{z} \mid z=1, \cdots, Z\right\}$. Different context models provide different multiscale modeling, the multi-objective problem in (8) is roughly analogous to the multiple criteria: $P_{a}, P_{b}$ and $P_{c}$. The above problem can be solved by a heuristic algorithm called the multistage problem-solving technique [11].

Both JMCMS [11] and HMT-3S [10] can improve segmentation results in terms of $P_{a}, P_{b}$ and $P_{c}$ by emphasizing the two terms in (4), respectively. It is shown in [12] that combination of them provides the best segmentation result regarding $P_{a}$ and $P_{b}$.

\subsection{Multiresolution Gaussian Autoregressive Models (MGAR) based on MMPM ${ }^{\text {[13] }}$}

A double-stochastic model is proposed in [13] for multiresolution textured-image segmentation where the observed image is represented as a multiresolution Gaussian autoregressive (MGAR) model and class labels are assumed to be dependent on both the same scale and the adjacent finer and coarser scales as a 3-D MRF.

The optimization criterion used for segmentation is the minimization of the expected value of the number of misclassified nodes in a multiresolution lattice. The estimator that satisfies this criterion is referred to as the "multiresolution maximization of the posterior marginals" (MMPM) estimator, and is a natural extension of the single-resolution MPM estimate [13]. The cost function in (1) is given by 


$$
C(X, x)=\sum_{j=0}^{J-1} \sum_{s \in S^{(j)}} \delta\left(X_{s}^{(j)}, x_{s}^{(j)}\right)
$$

Where $J$ denotes the total number of levels of the multiresolution lattice $S$, and the multiresolution representation of the observed image $Y$ and the class label pyramid $X$ are defined on the same lattice $S$. The segmentation $x$ that minimizes the conditional expectation of this cost function will be denoted as $x^{*}$. Thus

$$
\begin{aligned}
E\left[C\left(X, x^{*}\right) \mid Y=y\right] & =\min _{x} E\left[\sum_{s \in S}\left(1-\delta\left(X_{s}, x_{s}\right)\right) \mid Y=y\right] \\
& =\min _{x} \sum_{s \in S} E\left[\left(1-\delta\left(X_{s}, x_{s}\right)\right) \mid Y=y\right] \\
& =\min _{x} \sum_{s \in S} P\left(X_{s} \neq x_{s} \mid Y=y\right) \\
& \left.=\min _{x} \sum_{s \in S}\left(1-P\left(X_{s}=x_{s}\right) \mid Y=y\right)\right) \\
& =\max _{x} \sum_{s \in S} P\left(X_{s}=x_{s} \mid Y=y\right)
\end{aligned}
$$

where $\delta\left(X_{s}, x_{s}\right)=1$, if $X_{s}=x_{s} ; \delta\left(X_{s}, x_{s}\right)=0$, otherwise.

It is assumed that the number of distinct textures in the observed image is known in this approach, but the parameters $\theta$ of the MGAR model - the means, prediction coefficients, and prediction error variances of different textures are unknown. A modified version of the EM algorithm is used to estimate the parameters [13].

The Gibbs sampler with constant temperature can be used to generate a Markov chain $X(t)$ which converges in distribution to a random field with probability mass function $p_{X \mid Y}(X \mid Y, \theta)$. The marginal conditional probabilities $p_{X_{s} \mid Y}(k \mid Y, \theta)$, which are to be maximized, are then approximated as the fraction of time the Markov chain spends in state $k$ at node $s$, for each $k$ and $s$ [13]. If $N_{0}$ is the number of iterations (complete passes through the pyramid) of the Gibbs sampler, then the approximations

$$
p_{X_{s} \mid Y}(k \mid Y, \theta)=\frac{1}{N_{0}} \sum_{t=1}^{N_{0}} \delta\left(X_{s}(t), k\right) \quad \forall k, s
$$

provide estimates of the value needed to obtain the MMPM estimate of $X$. Integrating the multiresolution EM with the approximated MMPM algorithm, image segmentation and parameters estimation can be obtained simultaneously [13].

\subsection{Multi-grid Belief Propagation ${ }^{[14]}$}

Markov random field models provide a robust and unified framework for image segmentation, but the MRF framework yields an optimization problem that is NP hard, and it is usually highly advantageous to use graphs as diagrammatic representations to facilitate analysis and manipulations. One of the feasible approximation solutions is belief propagation [14-16]. Belief Propagation (BP) algorithm utilizes the conditional independent properties in the network to derive efficient solutions. Corresponding to the MPM and the MAP estimators, there are two types of BP algorithms. One is belief update (BU) also known 
as the sum-product algorithm for the MPM inferences, and another is belief revision (BR) a.k.a. the max-product algorithm for the MAP inferences [15].

The quality of image segmentation can be described in an energy function as following

$$
E(x)=\sum_{s \in S} D_{x_{s}}\left(x_{s}\right)+\sum_{(s, p) \in N} W\left(x_{s}, x_{p}\right)
$$

Where $N$ are the (undirected) edges in the four-connected image grid graph. $D_{x_{s}}\left(x_{s}\right)$ is the cost of assigning label $x_{s}\left(x_{s} \in\{1, \cdots, K\}\right)$ to pixel $s$ in the lattice $S$, and is referred to as the data cost. $W\left(x_{s}, x_{p}\right)$ measures the cost of assigning different labels to two neighboring pixels $s$ and $p$, and is generally referred to as the discontinuity cost. An intuitive measurement of the discontinuity cost is based on the degree of difference between the two neighboring labels, that is, $W\left(x_{s}, x_{p}\right)=V\left(x_{s}-x_{p}\right)$.

The BP algorithm works by passing messages around the graph defined by the fourconnected image grid. The method is iterative, with messages from all nodes (pixels) being passed in parallel. At each iteration, in the max-product BP algorithm, new messages can be computed with negative log probabilities where the max-product becomes a min-sum:

$$
m_{s \rightarrow p}^{t}\left(x_{p}\right)=\min _{x_{s}}\left(V\left(x_{s}-x_{p}\right)+D_{x_{s}}\left(x_{s}\right)+\sum_{q \in N(s) \backslash p} m_{q \rightarrow s}^{(t-1)}\left(x_{s}\right)\right)
$$

In the sum-product BP algorithm, new messages are updated as

$$
m_{s \rightarrow p}^{t}\left(x_{p}\right)=\min _{x_{s}}\left(V\left(x_{s}-x_{p}\right) \cdot D_{x_{s}}\left(x_{s}\right) \cdot \prod_{q \in N(s) \backslash p} m_{q \rightarrow s}^{(t-1)}\left(x_{s}\right)\right)
$$

The standard implementation of max-product message passing algorithm on the grid graph runs in $O\left(n K^{2} T\right)$ time, where $n$ is the number of pixels in the image, $K$ is the number of possible labels for each pixel and $T$ is the number of iterations. So the computation complexity is very high for images with large sizes.

Felzenszwalb and Huttenlocher [14] proposed an efficient multi-grid BP technique without changing the graph structure and the energy function: first the data costs are constructed from fine to coarse scales, where the data cost at coarse level can be calculated by summing over four data costs at the next finer level; then the message propagation process is started at the coarsest level, and the belief information is propagated from coarse to fine scales.

In contrast, the underlying graph in the example proposed in [16] is changed. First, a multiscale algebraic multigrid technique is used to select nodes that strongly influence others as the coarse nodes at the next coarser level, and an iterated weighted aggregation (IWA) process is followed to calculate the new edge weights among the selected coarse nodes. Second, given the belief propagation result at coarse level is interpolated as a start point for the next finer scale. Since the start point is believed to be close to the true solution, this $\mathrm{BP}$ is expected to converge rapidly.

\subsection{Multiscale probabilistic reasoning model ${ }^{[1]}$}

The quadtree structure induces causal properties that enable design of a non-iterative coarse-to-fine multiscale segmentation algorithm. However, an important disadvantage of the quadtree structure is that blocks that are spatial adjacent may not have common 
neighbors at the next coarser scale, and this may result in blocky artifacts in the final segmentation [1].

Fan and Xia listed five contextual models in [11], and proved that the context- 4 model can provide a high percentage of boundaries that coincide with the true ones. A hierarchical probabilistic reasoning model is proposed in [1] to alleviate the problem of blocky artifacts by providing a quadtree structure which combined with a spatial lattice neighborhood at each scale.

This hierarchical probabilistic reasoning model [1] is very different from the multiscale models proposed in [6-13]. The concept of scale in [1] is related to the size of a block in the spatial domain. While the scale described in [6-13] is related to either the Gaussian pyramid or the wavelet decomposition of the observed image. The Gaussian low-pass filter can alleviate texture noise to some degree, but it can also blur edges at the same time, especially for those low-contrast edges. The wavelet transform is very useful to represent the singularities (edges and ridges) in an image at multiple scale and three different orientations [8]. However, for textile images, the singularities are corrupted by uniformly distributed texture structure (see Fig.2). So it is more appropriate to perform segmentation in the spatial domain directly than in the wavelet-domain, and the experimental results in Section 3 also prove this scheme is inappropriate for textile images.

As we know that a large block usually enhances the classification reliability (because the use of many pixels can lessen the disturbance caused by texture noise), but simultaneously risks having pixels of different classes inside the block; while a small block reduces the possibility of having multiple classes in the block, but sacrifices classification reliability due to the paucity of color information and texture noise. So both the large and small scale behavior should be utilized to properly segment both large homogeneous regions and detailed boundary regions.

In [1], no special prior distribution assumption is made about the size and shape of regions. At every increasing scale, each block is subdivided into four child blocks, forming a quadtree structure. By adopting the context- 4 model proposed in [11] the correlations of spatial blocks across different scales and within the same spatial scale are integrated as shown in Fig.4

$$
\hat{x}^{(n)}=\underset{k}{\arg \max }\left\{p_{b^{(n)} \mid c_{k}}\left(b^{(n)} \mid c=c_{k}\right) \cdot p_{c_{k} \mid v_{b}}\left(c=c_{k} \mid v_{b}\right)\right\}
$$

Where $p_{b^{(n)} \mid c_{k}}\left(b^{(n)} \mid c=c_{k}\right)$ is the likelihood of the block $b^{(n)}$ at scale $n$ given the dominant color $c_{k}(k=1, \cdots, K)$. Since textile images are corrupted by non-Gaussian texture noise, it is not appropriate to specify this conditional probability $p_{b^{(n)} \mid c_{k}}\left(b^{(n)} \mid c=c_{k}\right)$ as a multivariate Gaussian distribution as done in other multiscale models [6-13]. In [1], we calculated the ratio of how many pixels in the current block $b^{(n)}$ are within a sphere centered at the dominant color $c_{k}(k=1, \cdots, K)$ with a radius of $r_{k}$ in the color space as the likelihood:

$$
p_{b^{(n)} \mid \mathbf{c}_{k}}\left(b^{(n)} \mid \mathbf{c}=\mathbf{c}_{k}\right)=\frac{\#\left(\left|c_{p_{i j}}-c_{k}\right|<r_{k}, p_{i j} \in b^{(n)}\right)}{\operatorname{size}\left(b^{(n)}\right)}
$$


Where \# is the counting operator, and $r_{k}$ is half of the minimum distance between the dominant color $c_{k}(k=1, \cdots, K)$ and the other dominant color $c_{l}(l \neq k, l=1, \cdots, K)$.
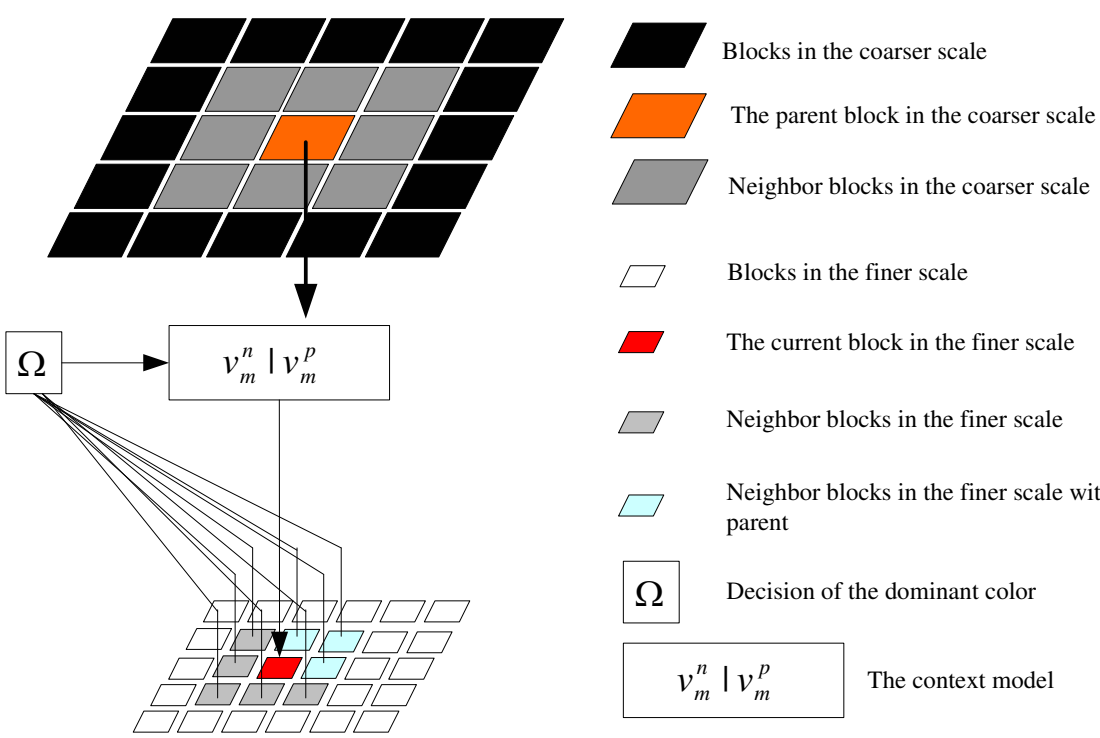

Fig. 4. Hierarchical probabilistic reasoning model [1]

The contextual information $v_{b}$ in (15) contains the color information of its parent block at the next coarser scale and that of the eight neighbor blocks of the current block at the same scale. We assume that the dependence on its parent block's color information is totally uncorrelated with the dependencies on its eight neighbor blocks' color information at the same scale. Therefore we can write the contextual probability $p_{c_{k} \mid v_{b}}\left(c=c_{k} \mid v_{b}\right)$ as

$$
p_{c_{k} \mid v_{b}}\left(c=c_{k} \mid v_{b}\right)=p\left(c=c_{k} \mid v_{b}^{\text {parent }}\right) \cdot p\left(c=c_{k} \mid v_{b}^{\text {neighbor }}\right)
$$

where "parent" denotes the parent block at the next coarser scale, and "neighbor" denotes the eight neighboring blocks at the same scale of the current block. According to Bayes rule, the probability $p\left(c=c_{k} \mid v_{b}^{\text {parent }}\right)$ or $p\left(c=c_{k} \mid v_{b}^{\text {neighbor }}\right)$ can be computed as follows:

$$
\begin{aligned}
p\left(c=c_{k} \mid v_{b}^{\tilde{b}}\right)= & \frac{p\left(v=v_{b}^{\tilde{b}} \mid c=c_{k}\right) p\left(c=c_{k}\right)}{p\left(v=v_{b}^{\tilde{b}}\right)} \\
= & \frac{p\left(v=v_{b}^{\tilde{b}} \mid c=c_{k}\right) p\left(c=c_{k}\right)}{\sum_{j=1}^{K} p\left(c=c_{j}\right) p\left(v=v_{b}^{\tilde{b}} \mid c=c_{j}\right)}
\end{aligned}
$$

where $\sim$ indicates either "parent" or "neighbor". The prior distribution of the dominant color $p\left(c=c_{k}\right)(k=1, \cdots, K)$ can be computed according to (16), where $b^{(n)}$ will be the size of 
the entire image. The likelihood $p\left(v=v_{b}^{\text {parent }} \mid c=c_{k}\right)$ of the parent block and the likelihood $p\left(v=v_{b}^{\text {neighbor }} \mid c=c_{k}\right)$ of the eight neighbor blocks, given the dominant colors $c_{k}(k=1, \cdots, K)$, can be computed from coarse to fine scales according to (15) respectively.

\section{Performances of multiscale image segmentation techniques on textile images}

In this section we focus on textile image segmentation based on several representative multiscale image segmentation techniques, and test their performance analytically.

Texture noise - the texture appearance of the fabric in textile images makes bottom-up, solely image-driven segmentation techniques always prone to errors [1]. Most of textile images are taken directly from the production line in textile industries, there are no ground truth segmentation results for these textile images, and it is difficult for us to manually prepare training data for training-based supervised image segmentation [7, 8]. In this paper we mainly consider supervised multiscale image segmentation, that is, it is assumed the number of distinct colors in the input textile image is known, and the dominant colors and their corresponding features can be obtained earlier.

The texture statistical characteristics are very helpful for image segmentation when the given image contains different visual texture regions [3, 8, 10-13]. However textile images contain uniformly distributed texture structure (as shown in Fig.1), so the color information is the main feature can be handled with during segmentation with during segmentation. Since there are usually only a few dominant colors in textile images, we obtain dominant colors by picking and averaging homogeneous regions from the given image. We extract six dominant colors: blue, red, white, yellow, dark green and shallow green from textile image 1, and eleven dominant colors from textile image 2 , and five dominant colors from textile image 3 and textile image 4, respectively.

In order to capture the cross-scale relationships of texture regions, prior to segmentation, learning-based supervised image segmentation techniques $[7,8]$ have to pick large enough homogeneous regions as training data from textile images. However, some of the dominant colors only occupied very small areas in textile images, such as black color in textile image 2 and textile image 3, so we only apply the HMTSeg algorithm [8] to textile image 1 and 4 because in these two images each dominant color occupies large enough area. In this paper we only examine supervised multiscale image segmentation techniques on textile images: the multiscale probabilistic reasoning model [1], sequential MAP [6], HMTSeg [8], belief propagation [14].

In the multiscale probabilistic reasoning model [1], no special prior distribution is assumed about the size and shape of regions, but in the SMAP [6] a multivariate Gaussian distribution is applied for that purpose, so in addition to inputting the number of dominant colors and corresponding RGB values, the SMAP algorithm requires inputting the covariance matrices of the three RGB channels of the dominant colors. The SMAP algorithm is good for textile images with fine texture structure and large homogeneous regions, such as textile image 4. Although the computation complexity of the SMAP algorithm is higher than that of the maximum likelihood algorithm, the performance of SMAP is comparable to that of ML for textile images with coarse fabric texture structure and delicate structures.

In order to apply the HMTSeg algorithm [8] to textile images, we have to pick several homogeneous blocks from the inputted image as training data. Both textile image 1 and 
textile image 4 have large homogeneous regions, and for each dominant color, we randomly pick ten $16 \times 16$ blocks. With this training data and interscale tying in the wavelet-domain HMT models [8], the EM training algorithm [9] is used to estimate the parameters of the HMT model for each dominant color. Since each HMT model is trained on $16 \times 16$ uniform blocks, the output segmentation results are very blocky (see Fig.5. (f) and Fig.8. (f)). Though the wavelet-domain HMT model is very helpful to images with edges and ridges, in textile images, edges and ridges are corrupted by uniformly distributed texture noise and the test image generally is much larger than the training images, so repeat the likelihood computations for image subblocks assuming that the blocks are independent. The waveletdomain HMT model which designed for distinct visual texture statistics is not suitable for textile images, and from the segmentation results, we can see that the misclassified color areas are large. We don't apply the HMTSeg algorithm to textile image 2 and 3 because some of the dominant colors only occupied very small areas in these two images, and it is difficult for us to pick large enough training blocks from those images.

The belief propagation approach [14] can be used to approximate the MAP solutions to MRF problems. The local minima found by BP are minima over "large neighborhoods", so they can produce high accurate segmentation results in practice. In [14] they proposed three algorithmic schemes to improve the running time of the loopy belief propagation approach: the min-convolution algorithm, belief propagation on grid graphs and a multi-grid method which speed up and reduce the memory requirements of belief propagation. In order to allow for large discontinuities in the labelling, the cost function stops growing after the difference becomes large, and they introduced a data term $d$ to $V(x)$ in eq. (13) to control the cost to stop increasing when the discontinuities are large. Another parameter $\lambda$ is applied to balance between the fidelity of the data cost term $D(x)$ and the smoothness of the discontinuity cost $V(x)$. Both $d$ and $\lambda$ are selected heuristically. In all the experiments, $d$ is set as $10^{4}$, and $\lambda$ is set as 15 . The iteration number for each scale is set as 10 , and the experimental results do not change too much as the iteration number increases above 10 . The BP algorithm is good for large homogeneous regions but will simplify fine structures, and good examples are illustrated in Fig.6 and Fig.8.

\section{Conclusions}

In contrast with natural images, textile images have some very distinctive properties: 1) generally there are a few dominant colors in a textile image, whereas there may exist hundreds of significant colors in a natural image; 2) the fabric texture structure caused by the color halftoning technique in textile printing has a great influence on the colors' appearance in textile images.

The uniform fabric structure (texture noise) makes it a difficult problem for the existing automatic color segmentation methods to extract the dominant colors from textile images. In this chapter, we study various multiscale image segmentation techniques by considering image segmentation as inferring the "best" labeling configuration $X$ from the observed image data $Y$. The causal properties induced by multiscale structure enable the design of exact, non-iterative inference algorithms. In most multiscale image segmentation techniques, the joint probability density of $(X, Y)$ and the marginal density are modeled as multivariate Gaussian distributions or Gaussian mixtures. Whereas textile images are corrupted by non- 
Gaussian texture noise, it is difficult to specify a prior distribution about the size and shape of regions, and this is an open problem of our future research work.

We analyze the performances of different multiscale image segmentation techniques on some representative textile images, and find that the spatial contextual model proposed in [1] can produce high visual quality segmentation results on average. Since these textile images have no ground truth segmentation results, it is difficult for us to evaluate the performances of different techniques quantitatively. So to evaluate the segmentation results quantitatively is also a problem of our future research works.

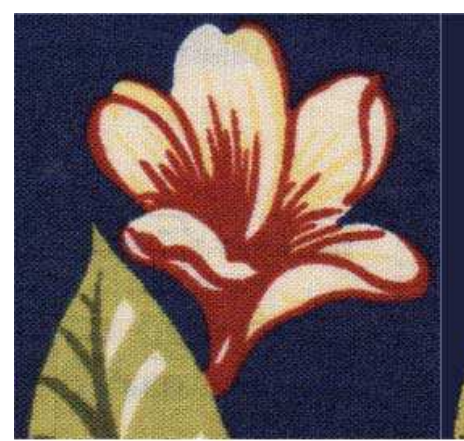

(a) Textile image 1

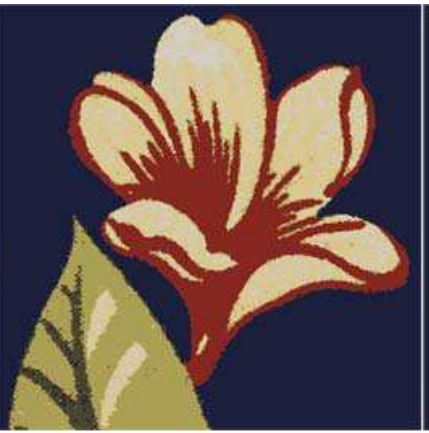

(b) Probabilistic Reasoning [1]

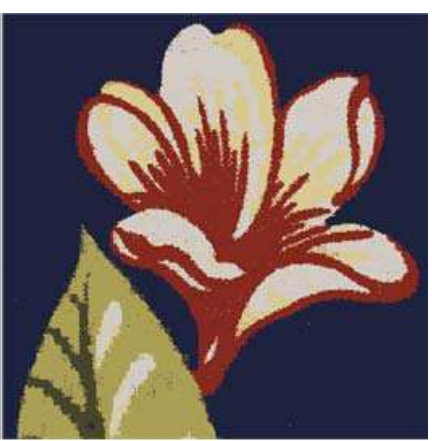

(c) SMAP [6]

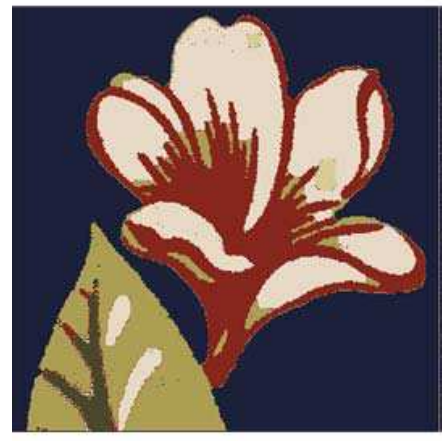

(d) BP $[14]$

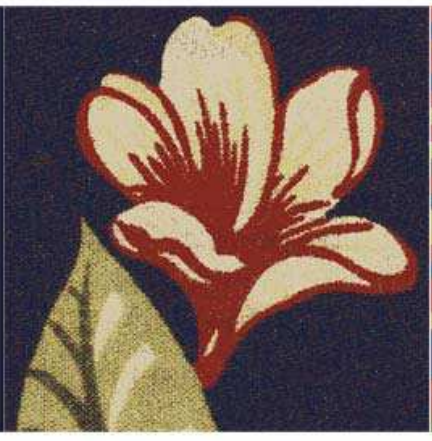

(e) ML

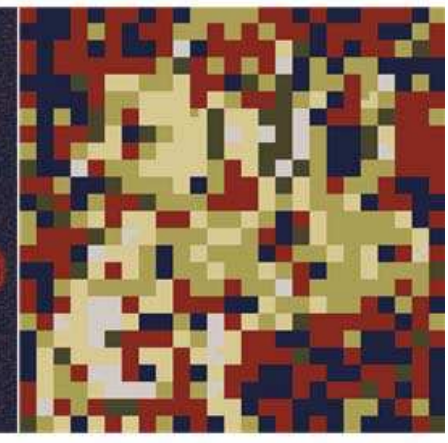

(f) HMT [8]

Fig. 5. Segmentation results of textile image 1 


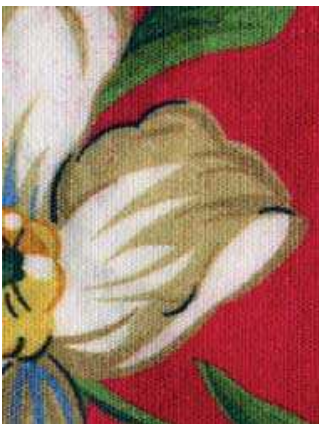

(a) Textile image 2

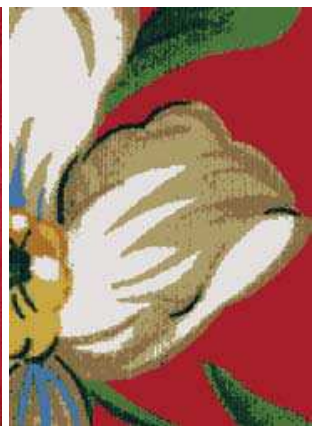

(b) Probabilistic Reasoning [1]

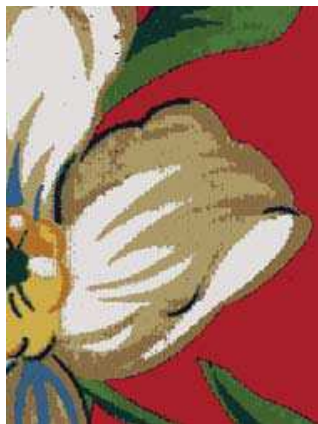

(c) SMAP [6]

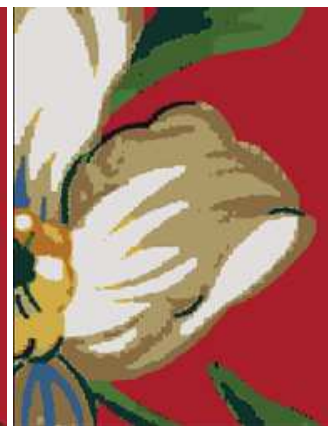

(d) BP [14]

Fig. 6. Segmentation results of textile image 2

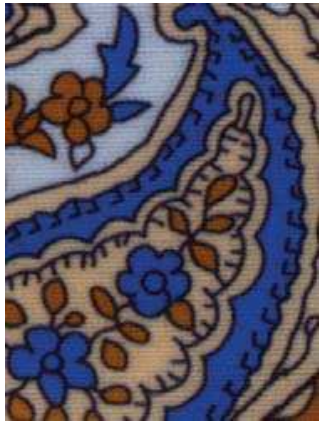

(a) Textile image 3

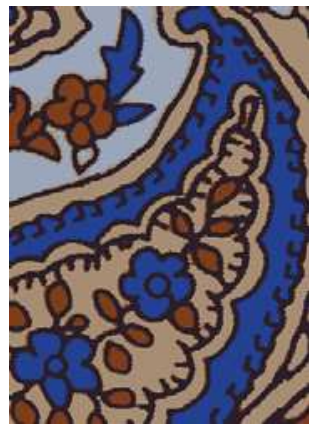

(b) Probabilistic Reasoning [1]

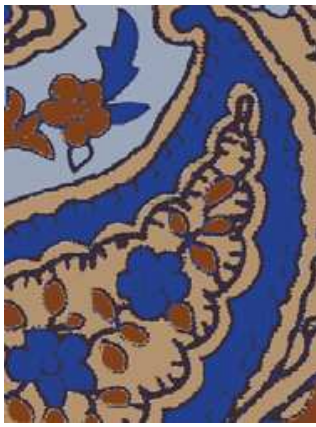

(c) SMAP [6]

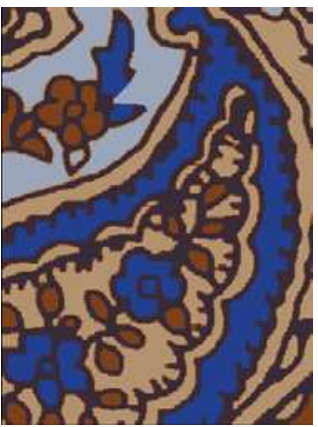

(d) BP [14]

Fig. 7. Segmentation results of textile image 3 


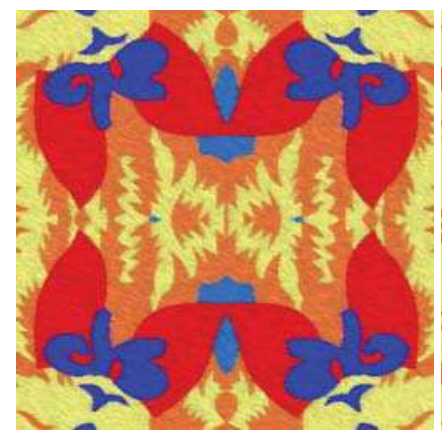

(a) Textile image 1

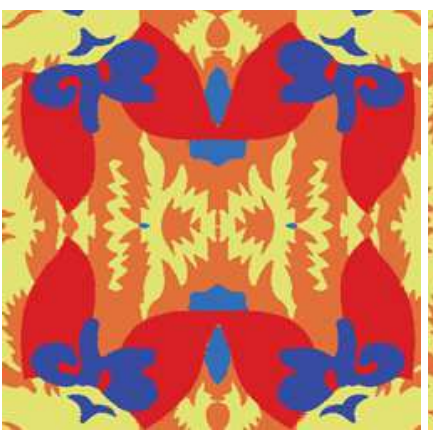

(b) Probabilistic Reasoning [1]

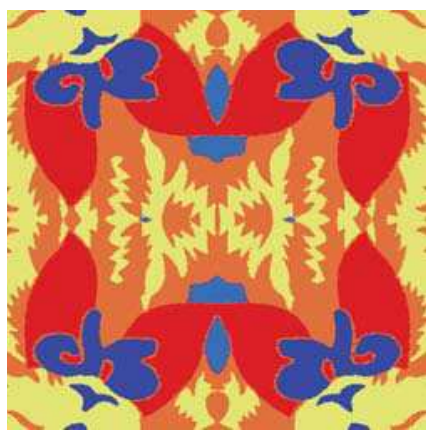

(c) SMAP [6]

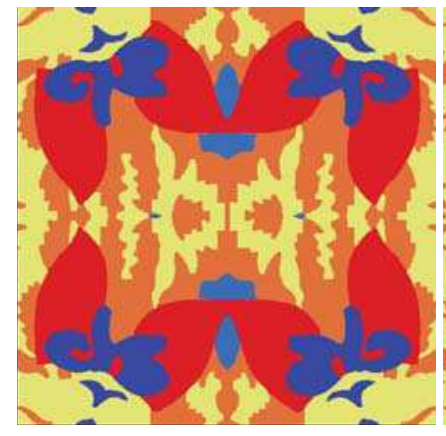

(d) BP [14]

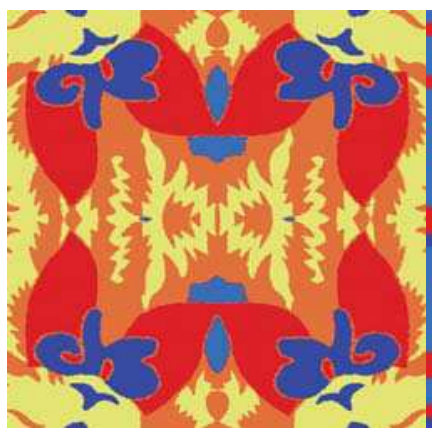

(e) ML

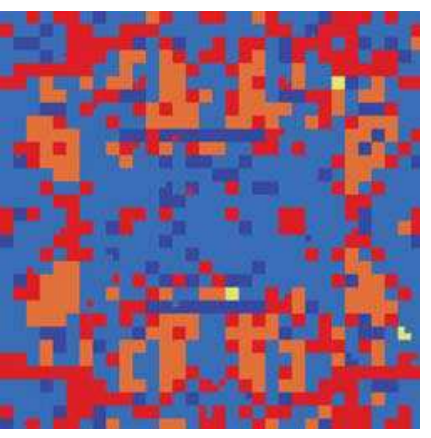

(f) HMT [8]

Fig. 8. Segmentation results of textile image 4

\section{References}

[1] $\mathrm{Lu}$, X.-Q. (2007). Color textile image segmentation based on multiscale probabilistic reasoning, Optical Engineering, 46(8), Article ID 087002

[2] Lu, X.-Q.; Sakaino, H. (2009). A spatial adaptive filter for smoothing of non-Gaussian texture noise, Proc. of IEEE ICASSP, pp.841-844, Taibei, Apr. 2009, IEEE

[3] Bouman, C.; Liu, B. (1991). Multiple resolution segmentation of textured images, IEEE Trans. On Pattern Analysis and Machine Intelligence, vol.13, no.2, pp.99-113, 1991

[4] Wilsky, A. S. (2002). Multiresolution Markov models for signal and image processing, Proc. of the IEEE, vol.90, no.8, pp.1396-1458, 2002

[5] Laferte, J.-M.; Heitz, F. (2000). Discrete Markov image modeling and inference on the quadtree, IEEE Trans. On Image Processing, vol.9, no.3, pp.390-404, 2000

[6] Bouman, C. A.; Shapiro, M. (1994). A multiscale random field model for Bayesian image segmentation, IEEE Trans. On Image Processing, vol.3, no.2, pp.162-177, 1994

[7] Cheng, H.; Bouman, C. A. (2001). Multiscale Bayesian segmentation using a trainable context model, IEEE Trans. On Image Processing, vol.10, no.4, pp.511-525, 2001 
[8] Choi, H.; Baraniu, R. G. (2001). Multiscale image segmentation using wavelet-domain hidden Markov models, IEEE Trans. On Image Processing, vol.10, no.9, pp.13221331, 2001

[9] Crouse, M. S.; Nowak, R. D., and Baraniu, R. G. (1998). Wavelet-based statistical signal processing using hidden Markov models, IEEE Trans. On Signal Processing, vol.46, no.4, pp.886-902, 1998

[10] Fan, G.; Xia, X.-G. (2000). Maximum likelihood texture analysis and classification using wavelet-domain hidden Markov models, Proc. of 34th Asilomar Conference on Signals, Systems and Computers, Pacific Grove, CA, 2000

[11] Fan, G.; Xia, X.-G. (2001). A joint multicontext and multiscale approach to Bayesian Image segmentation, IEEE Trans. On Geoscience and Remote Sensing, vol.39, no.12, pp. 2680-2688, 2001

[12] Fan, G.; Xia, X.-G. (2003). Wavelet-based texture analysis and synthesis using hidden Markov models, IEEE Trans. On Circuits and Systems - I: Fundamental Theory and Applications, vol.50, no.1, pp. 106-120, 2003

[13] Cormer, M. L.; Delp, E. J. (1999). Segmentation of textured images using a multiresolution Gaussian autoregressive model, IEEE Trans. On Image Processing, vol.8, no.3, pp.408-420, 1999

[14] Felzenszwalb, P. F.; Huttenlocher, D. P. (2006). Efficient belief propagation for early vision, International Journal of Computer Vision, vol.70, no.1, pp.41-54, 2006

[15] Weiss, Y.; Freeman, W. T. (2001). On the optimality of solutions of the max-product belief-propagation algorithm in arbitrary graphs, IEEE Trans. On Information Theory, vol.47, no.2, pp.736-744, 2001

[16] Xiong, L; Wang, F. \& Zhang, C. (2007). Multilevel belief propagation for fast inference on Markov random fields, Proc. of 7 th IEEE International Conference on Data Mining, pp.371-380, 2007 


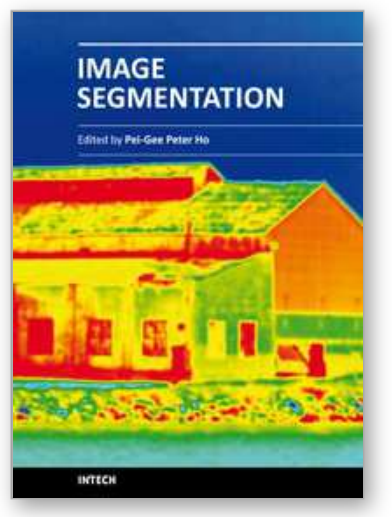

\author{
Image Segmentation \\ Edited by Dr. Pei-Gee Ho
}

ISBN 978-953-307-228-9

Hard cover, 538 pages

Publisher InTech

Published online 19, April, 2011

Published in print edition April, 2011

It was estimated that $80 \%$ of the information received by human is visual. Image processing is evolving fast and continually. During the past 10 years, there has been a significant research increase in image segmentation. To study a specific object in an image, its boundary can be highlighted by an image segmentation procedure. The objective of the image segmentation is to simplify the representation of pictures into meaningful information by partitioning into image regions. Image segmentation is a technique to locate certain objects or boundaries within an image. There are many algorithms and techniques have been developed to solve image segmentation problems, the research topics in this book such as level set, active contour, AR time series image modeling, Support Vector Machines, Pixon based image segmentations, region similarity metric based technique, statistical ANN and JSEG algorithm were written in details. This book brings together many different aspects of the current research on several fields associated to digital image segmentation. Four parts allowed gathering the 27 chapters around the following topics: Survey of Image Segmentation Algorithms, Image Segmentation methods, Image Segmentation Applications and Hardware Implementation. The readers will find the contents in this book enjoyable and get many helpful ideas and overviews on their own study.

\title{
How to reference
}

In order to correctly reference this scholarly work, feel free to copy and paste the following:

Xiqun Lu (2011). Multiscale Segmentation Techniques for Textile Images, Image Segmentation, Dr. Pei-Gee Ho (Ed.), ISBN: 978-953-307-228-9, InTech, Available from: http://www.intechopen.com/books/imagesegmentation/multiscale-segmentation-techniques-for-textile-images

\section{INTECH}

open science | open minds

\section{InTech Europe}

University Campus STeP Ri

Slavka Krautzeka 83/A

51000 Rijeka, Croatia

Phone: +385 (51) 770447

Fax: +385 (51) 686166

www.intechopen.com

\section{InTech China}

Unit 405, Office Block, Hotel Equatorial Shanghai

No.65, Yan An Road (West), Shanghai, 200040, China

中国上海市延安西路65号上海国际贵都大饭店办公楼405单元

Phone: +86-21-62489820

Fax: +86-21-62489821 
(C) 2011 The Author(s). Licensee IntechOpen. This chapter is distributed under the terms of the Creative Commons Attribution-NonCommercialShareAlike-3.0 License, which permits use, distribution and reproduction for non-commercial purposes, provided the original is properly cited and derivative works building on this content are distributed under the same license. 